\title{
Complete Blood Count Collection Tube
}

National Cancer Institute

\section{Source}

National Cancer Institute. Complete Blood Count Collection Tube. NCI Thesaurus. Code C113676.

A blood collection tube that contains the anti-coagulant EDTA, designed for complete blood counts and blood films. 